\title{
Characteristics and toxicity assessment of electrospun gelatin/PCL nanofibrous scaffold loaded with graphene in vitro and in vivo
}

This article was published in the following Dove Press journal: International Journal of Nanomedicine

\author{
Xi Chen* \\ Bei Feng* \\ Di-Qi Zhu \\ Yi-Wei Chen \\ Wei Ji \\ Tian-Ji Ji \\ Fen Li
}

Department of Cardiology, Shanghai Children's Medical Center, School of Medicine, Shanghai Jiaotong University, Shanghai, People's Republic of China

*These authors contributed equally to this work
Correspondence: Fen $\mathrm{Li}$

Department of Cardiology, Shanghai

Children's Medical Center, School of

Medicine, Shanghai Jiaotong University,

No.1678 Dongfang Road, Shanghai,

People's Republic of China

Tel +86213861 6161

Fax +862138616161

Email lifen@scmc.com.cn
Background: Electrospun gelatin/polycaprolactone (Gt/PCL) nanofibrous scaffolds loaded with graphene are novel nanomaterials with the uniquely strong property of electrical conductivity, which have been widely investigated for their potential applications in cardiovascular tissue engineering, including in bypass tracts for atrioventricular block.

Purpose: Electrospun Gt/PCL/graphene nanofibrous mats were successfully produced. Scanning electron micrography showed that the fibers with graphene were smooth and homogeneous. In vitro, to determine the biocompatibility of the scaffolds, hybrid scaffolds with different fractions of graphene were seeded with neonatal rat ventricular myocytes. In vivo, Gt/PCL scaffolds with different concentrations of graphene were implanted into rats for 4,8 and 12 weeks.

Results: CCK-8 assays and histopathological staining (including DAPI, cTNT, and CX43) indicated that cells grew and survived well on the hybrid scaffolds if the mass fraction of graphene was lower than $0.5 \%$. After implanting into rats for 4,8 or 12 weeks, there was no gathering of inflammatory cells around the nanomaterials according to the HE staining results.

Conclusion: The results indicate that Gt/PCL nanofibrous scaffolds loaded with graphene have favorable electrical conductivity and biological properties and may be suitable scaffolds for use in the treatment of atrioventricular block. These findings alleviate safety concerns and provide novel insights into the potential applications of Gt/PCL loaded with graphene, offering a solid foundation for comprehensive in vivo studies.

Keywords: gelatin, polycaprolactone, graphene, electrical conductivity, toxicity, atrioventricular block

\section{Introduction}

Atrioventricular conduction block (AVB) is a serious clinical problem. ${ }^{1,2}$ Currently, other than permanent pacemaker placement, there are no feasible therapies for this disease. $^{3,4}$ Although the effectiveness of cardiac pacemaker implantation as palliative treatment seems irrefutable, there is less optimism regarding its long-term efficacy, especially in children. ${ }^{5}$ There is thus an important clinical need to explore innovative, sustainable, and effective treatments for AVB that are not dependent on an artificial cardiac pacemaker.

Cardiac tissues require specific contractile properties that are directly related to cellular orientation and elongation. ${ }^{6-8}$ It remains a great challenge to engineer cardiac tissues that simulate the microarchitecture of the native myocardium, in 
terms of topographical features and biophysical and mechanical properties. Recently, significant efforts have been made to engineer cardiac tissue for synthetic transplants to replace damaged tissue. ${ }^{9-11}$ The biomaterials currently used in cardiac tissue engineering have limitations, including a lack of electrical conductivity and appropriate mechanical properties; both of these parameters have key roles in regulating cardiac cell behavior. ${ }^{11}$

Electrospinning is a well-established and inexpensive technique for fabricating nanofiber scaffolds, and has been applied in various fields including biosensors, wound healing, drug delivery, and tissue engineering. ${ }^{12,13}$ In our previous studies, we examined the utility of electrospun gelatin/polycaprolactone (Gt/PCL) nanofibrous scaffolds as biomimetic materials in tissue engineering, for applications such as regeneration of vascular tissue and cartilage repairs. $^{14-18}$ PCL, a semi-crystalline hydrophobic linear polymer, is known for its good mechanical strength, biocompatibility, and ease of spinnability. Gelatin, which is derived from partial hydrolysis of collagen, has low immunogenicity, high biocompatibility, and commercial availability at low cost, all of which facilitate its widespread applications in pharmaceutical and medical fields. PCL could be combined with gelatin in order to yield a nanofibrous mat with highly improved mechanical, physical, and biological properties. ${ }^{19,20}$

Theoretically, AVB patients need a bypass tract rebinding the electrical conduction from atrium to ventricle. The main challenge is the construction of a linking tract that can transmit the electrical signals generated by the sinoatrial node. Even though former electrospun Gt/PCL nanofibrous scaffolds showed some electrical property, it could not achieve the required electrical conductivity. ${ }^{19}$ Graphene is a novel single-layer two-dimensional $\mathrm{sp}^{2}$ carbon nanomaterial in a honeycomb structure, which shows outstanding electrical conductivity and biological characteristics. $^{21,22}$ Graphene-based materials have also emerged as ubiquitous nanomaterials in various biomedical applications, such as biosensing, bioimaging, gene and drug delivery, cancer therapy, and tissue engineering, owing to their remarkable electrical, optical, chemical, thermal, and mechanical properties. ${ }^{22-26}$ Several recent studies indicate that graphene is a promising reinforcing material for regulating cardiac cell behavior, owing to its excellent physical properties such as high mechanical strength and unique electrical conductivity. ${ }^{27-29}$ In the current study, we generated electrospun Gt/PCL/graphene nanofibrous mats, which showed superb electrical conductivity, suitable for use as a bypass tract for electrical conduction. However, environmental issues are of significant concern, since graphene is toxic to cardiac cell lines and animals, and biosafety levels need to be established. Toxicological assessment of graphene, which depends on the dose used, the characteristics of the nanomaterial, and the exposure concentration and time, must be performed prior to its use in biomedical applications. ${ }^{30,31}$ Therefore, electrospun Gt/PCL/graphene cannot be universally defined as toxic or non-toxic, and requires individual assessment in vitro and in vivo. Most importantly, we need to assess the toxicity of different types of nanomaterials to the organism at the dose required to achieve their electrical conducting effects. Data from the current study provide valuable information supporting the potential application of electrospun Gt/PCL/graphene nanoparticles for rebinding AVB patients in the clinic.

\section{Materials and methods}

\section{Fabrication of electrospun Gt/PCL/ graphene nanoparticles}

Gt/PCL membranes loaded with graphene were fabricated by electrospinning, as previously described. In brief, polymers of gelatin type A (300 Bloom) from porcine skin in powder form and PCL (molecular weight 80,000) were obtained from Sigma-Aldrich (St Louis, MO, USA), and graphene was obtained from XFNANO (China). Then, gelatin, PCL and graphene were dissolved separately in hexafluoroisopropanol (HFIP) at a concentration of $10 \mathrm{wt}$ $\%$. Before electrospinning, the gelatin and PCL were mixed in a 50:50 (v:v) ratio with a trace amount of acetic acid (acetic acid/HFIP $0.2 \%$ ) and the graphene was separately dispersed in HFIP. Then they were stirred vigorously at room temperature for $72 \mathrm{~h}$. The electrospinning conditions were as follows: $2.0 \mathrm{~mL} / \mathrm{h}$ injection rate, $15 \mathrm{kV}$ voltage, and $12 \mathrm{~cm}$ distance between the syringe nozzle and the grounded aluminum sheet $\left(200 \times 200 \mathrm{~mm}^{2}\right)$. Using a flat plate as a receiving device, different mass fractions $(0.0 \%, 0.3 \%, 0.5 \%, 0.8 \%$, and $1.0 \%)$ of disordered Gt/ PCL membranes loaded with graphene were prepared by adjusting the spinning process. The Gt/PCL membranes loaded with graphene were vacuum dried for 1 week at room temperature. The membranes were lyophilized in a vacuum freeze-drier (Freezone 2.5L Triad; Labconco, Kansas City, MO, USA) for $24 \mathrm{~h}$. Eventually, the Gt/ PCL membranes loaded with graphene were sterilized for $0.5 \mathrm{~h}$ by ultraviolet irradiation for subsequent use. 


\section{Characterization of scaffolds}

The morphology of the electrospun Gt/PCL/graphene was observed by scanning electron microscopy (SEM; JSM5600LV, JEOL Ltd, Tokyo, Japan) at an acceleration voltage of $8-10 \mathrm{kV}$. Fiber diameters were measured using the ImageJ 1.40G software (National Institutes of Health, Bethesda, MD, USA). At least 100 nanofibers from each sample were analyzed in the various SEM images.

The mechanical properties of the electrospun fibrous membranes were determined using a tabletop uniaxial material testing machine (Instron-3343, Norwood, USA) equipped with a $50 \mathrm{~N}$ load cell. Rectangular specimens (30 mm $\times 10 \mathrm{~mm} \times 0.10-0.20 \mathrm{~mm}$ ) were stretched at a constant cross-head speed of $10 \mathrm{~mm}$ per minute. Five samples from each group were tested. For each specimen, the greatest slope in the linear region of the stress-strain curve, corresponding to a strain of $0-20 \%$, was used to calculate the Young's modulus.

The electrical conductivities of the membranes were determined using an electrical characteristic tester (Keithley 2400 Source Meter, USA) with a test voltage of $2.1 \mathrm{~V}$. Specimens were rectangular in shape $(50 \mathrm{~mm} \times 10 \mathrm{~mm})$, and five samples from each group were tested.

\section{Cell isolation and culture}

Neonatal rat ventricular cardiomyocytes (NRVCMs) were isolated and cultured according to previously established methods. Briefly, NRVCMs were harvested, centrifuged, washed, and then resuspended onto culture dishes at a cell density of $5 \times 10^{5}$ nucleated cells $/ \mathrm{cm}^{2}$ in Dulbecco's Modified Eagle's Medium (Hyclone, Logan, UT, USA) containing $10 \%$ fetal bovine serum (Bioind, Beit HaEmek, Israel), $100 \mathrm{U} / \mathrm{mL}$ penicillin, and $100 \mathrm{U} / \mathrm{mL}$ streptomycin (Hyclone). When primary cells reached more than $80-90 \%$ confluence, the cells were digested with $0.25 \%$ trypsin plus $0.02 \%$ ethylene diamine tetraacetic acid (Gibco, Waltham, MA, USA) and then subcultured at a cell density of $2 \times 10^{4}$ cells $/ \mathrm{cm}^{2}$. The electrospun $\mathrm{Gt} /$ $\mathrm{PCL} /$ graphene membranes were trimmed into round shapes with diameters of $1.5 \mathrm{~cm}$ (equal to the size of each well in a 24-well plate) and placed into 24-well plates.

\section{Cell apoptosis assay and biocompatibility evaluation of the scaffolds}

To study cell viability and apoptosis on the various substrates, NRVCMs were also seeded onto the scaffolds in 24-well plates at a density of $2 \times 10^{4}$ cells/well and quantified using a Cell Counting Kit-8 (CCK-8, Dojindo Molecular Technologies Inc, Rockville, MD, USA). At desired time points, the cell-seeded scaffolds were incubated in $10 \%$ CCK- 8 at $37{ }^{\circ} \mathrm{C}$ for $2 \mathrm{~h}$ in $5 \% \mathrm{CO}_{2}$. The absorbance of each well was measured at $450 \mathrm{~nm}$ using a microplate reader (Multiskan MK3, Thermo Electron Corporation, MA, USA). At least six samples were measured at each time point. After that, the remaining samples were treated in the same way and examined every other day. Cell viability was calculated as a percentage of the treated samples relative to untreated controls.

Different mass fractions $(0.0 \%, 0.3 \%, 0.5 \%, 0.8 \%$, and $1.0 \%$ ) of disordered Gt/PCL membranes loaded with graphene were assessed by immunohistochemical staining of the cell-scaffold constructs, including DAPI for nucleic acid staining, and specific markers of cardiomyocytes, such as cTNT and CX43. After that, the remaining samples were treated in the same way and examined every other day.

\section{Cell adhesion and living condition on $\mathrm{Gt} /$ $\mathrm{PCL} /$ graphene membranes}

To determine the cytocompatibility of Gt/PCL/graphene membranes, the morphology and adhesion of NRVCMs on Gt/PCL/graphene membranes were observed by SEM. The myocardial cells were seeded onto Gt/PCL/graphene membranes at a density of $2 \times 10^{4}$ cells/well in 24 -well plates ( 2 $\mathrm{cm}^{2}$ per well). Five days after cell seeding, samples were fixed with $2.5 \%$ glutaraldehyde overnight at $4{ }^{\circ} \mathrm{C}$. Samples were rinsed and dehydrated with graded concentrations $(50 \%, 70 \%, 80 \%, 90 \%, 95 \%$, and $100 \% \mathrm{v} / \mathrm{v})$ of ethanol. Subsequently, the samples were critical-point dried and examined by SEM.

\section{Implantation of scaffold constructs and gross observation}

All experimental procedures were approved by the Experimental Animal Use and Management Committee in Experimental Animal Research Institute, Shanghai Children's Medical Center affiliated to Shanghai Jiaotong University and with approved institutional protocols set by the China Association of Laboratory Animal Care. The membranes were cut into rectangles $(2 \mathrm{~cm} \times 7.5 \mathrm{~cm})$ with an average thickness of $20 \mu \mathrm{m}$, lyophilized in a Virtis Benchtop 6.6 vacuum freeze-dryer (SP Industries, Gardiner, NY, USA) for $24 \mathrm{~h}$, and sterilized for $30 \mathrm{~min}$ 
under ultraviolet radiation. The scaffold constructs were then implanted into rats (obtained from the Shanghai Laboratory Animal Center, Chinese Academy of Sciences, People's Republic of China), which had been anesthetized using $2.5 \%$ chloral hydrate. The skin of the dorsum was cut at approximately $2 \mathrm{~cm}$ by dissecting one side of the subcutaneous fascia, and the membranes were implanted subcutaneously. Six weeks after implantation, the mice were euthanized and the membrane constructs were dissected for further investigation. Each construct was observed grossly for shape, color, and size. For the long-term toxicity assessment, all surviving rats were carefully observed and weighed over the subsequent 4,8 and 12 weeks.

\section{Histological findings}

Following gross examination, samples were fixed in $4 \%$ paraformaldehyde, embedded in paraffin, and sectioned into 5- $\mu \mathrm{m}$ sections. The sections were prepared for histological analysis by staining with hematoxylin and eosin and Masson's trichrome.

\section{Statistical analysis}

All data presented are expressed as mean \pm standard deviation. Statistical analysis was carried out using single-factor analysis of variance. A value of $p<0.05$ was considered statistically significant.

\section{Results}

\section{Morphology and mechanical properties of the nanofibrous scaffold}

Composite Gt/PCL scaffolds with different dosage of graphene were prepared using the electrospinning method described above. As shown in the SEM images in Figure $1 \mathrm{~A}$, the nanofibers of pure Gt/PCL, Gt/PCL with $0.3 \%$ and $0.5 \%$ graphene had a uniform and smooth appearance; however, many beads could be detected in the nanofibrous scaffold of Gt/PCL with $0.8 \%$ or $1.0 \%$ graphene, indicating that the large amounts of graphene used might not have been fully dissolved, affecting the stability of the nanofibers. As shown in Figure 1B, the mean fiber diameters of the five scaffolds were about $489 \pm 68 \mathrm{~nm}, 551$ $\pm 71 \mathrm{~nm}, 578 \pm 91 \mathrm{~nm}, 579 \pm 96 \mathrm{~nm}$ and $595 \pm 119 \mathrm{~nm}$ (ImageJ). The mechanical properties of the scaffolds are particularly important because of the forces they endure in vivo after implantation. The Young's moduli were determined to be $28.74 \pm 3.35 \mathrm{MPa}, 31.68 \pm 4.04 \mathrm{MPa}, 32.44$ $\pm 3.68 \mathrm{MPa}, 32.97 \pm 6.11 \mathrm{MPa}$, and 37.20 $\pm 6.37 \mathrm{MPa}$ for the pure Gt/PCL, Gt/PCL with $0.3 \%, 0.5 \%, 0.8 \%$ and $1.0 \%$ graphene membranes, respectively (Figure 1C). As shown in Figure 1D, electrical conductivities of the membranes were determined to be $0.15 \pm 0.09,3.75 \pm 0.41,7.72$ $\pm 0.62,8.94 \pm 0.76$ and $11.15 \pm 1.04\left(10^{-3} \mathrm{~S} \cdot \mathrm{cm}^{-1}\right)$. Together, these results indicate that composite gelatin/PCL scaffolds with different dosages of graphene were successfully engineered.

\section{Apoptosis and morphology of nrvems on the scaffolds}

A CCK-8 test was carried out to observe the cell viability of NRVCMs on different scaffolds and on the tissue culture plate and all results were showed in Figure 2. Altogether, there were five groups, comprising Gt/PCL with $0.3 \%, 0.5 \%, 0.8 \%$, and $1.0 \%$ mass fractions of graphene, and pure Gt/PCL as a control. NRVCMs survived well on the first day regardless of the mass fraction of graphene, but the number of cells decreased along with culture time on the substrates with $0.8 \%$ and $1.0 \%$ graphene. On the fifth day of culture, the primary myocardial cell viability of Gt/PCL with a $0.8 \%$ fraction of graphene decreased to $74.36 \%$, compared with $68.62 \%$ for the $1.0 \%$ fraction of graphene, indicating that when the mass fraction was above $0.5 \%$, the scaffolds were toxic to myocardial cells. On the contrary, no obvious differences could be detected among the pure Gt/PCL and Gt/PCL with $0.3 \%$ and $0.5 \%$ fractions of graphene. On the ninth day of coculture, the cell viabilities for the $0.8 \%$ and $1.0 \%$ fraction of graphene decreased to $37.33 \%$ and $35.69 \%$, respectively, which indicated that the apoptosis rate of cells increased with the passage of time. However, the cell viabilities of the control group and Gt/PCL with graphene fractions of $0.3 \%$ and $0.5 \%$ remained high. Together, these results indicate that the Gt/PCL with a mass fraction of graphene lower than $0.5 \%$ had good biocompatibility with myocardial cells, providing further evidence of their potential applications in tissue engineering given their unique property of strong conductivity in cardiovascular tissue.

Cell adhesion and living conditions are critical parameters when evaluating whether a scaffold is biocompatible and suitable for cell culture. SEM was used to observe cell morphology and contacts between the cells and scaffolds. As shown in Figure 3, three different groups were studied: pure Gt/PCL, Gt/PCL with a graphene fraction of 
A

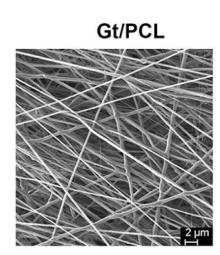

B
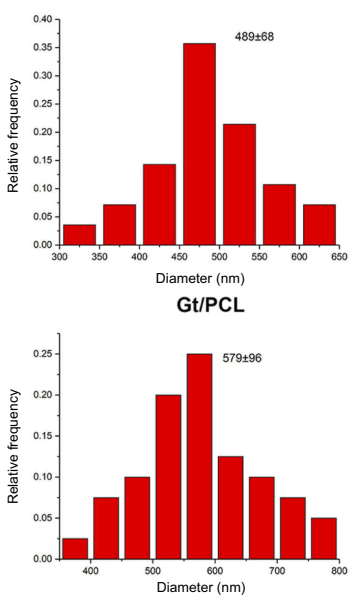

$0.8 \%$

C

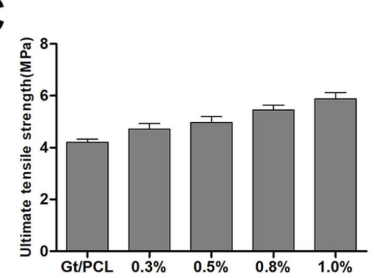

$0.8 \%$

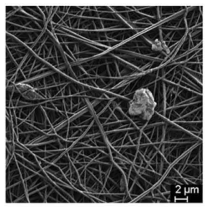

$1.0 \%$

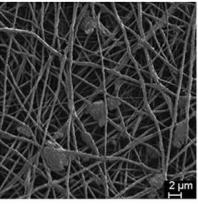

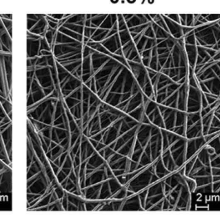

Diameter
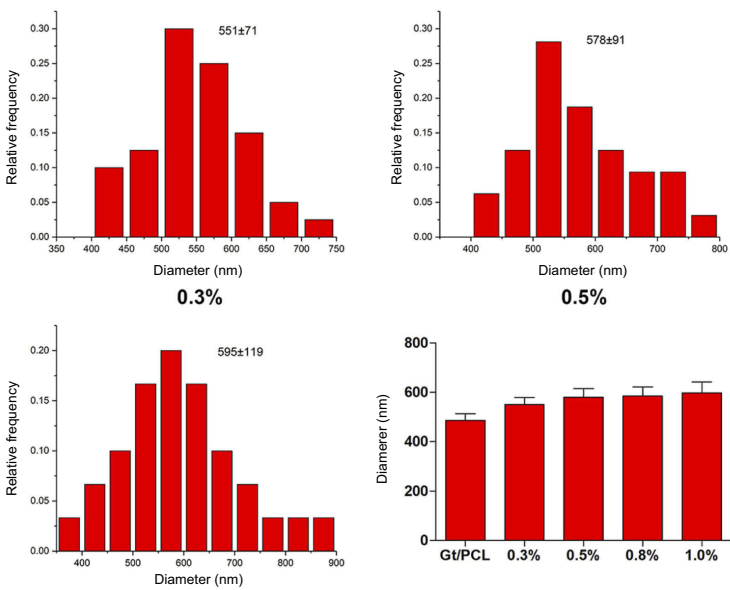

$1.0 \%$

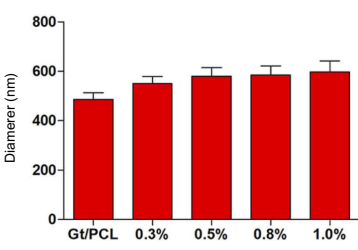

Diameters

\section{Strength}
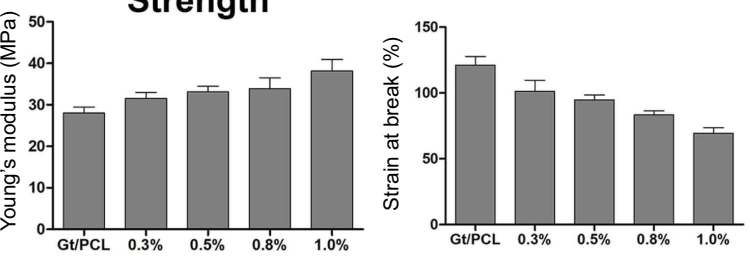

D

Conductivity

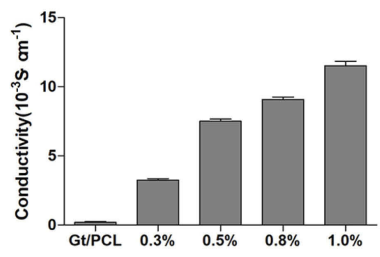

Figure I Characteristics of Gt/PCL/graphene nanofibrous scaffolds. (A) SEM images of Gt/PCL/graphene nanofibrous scaffolds, scale bar: 2 um; (B) Diameter distributions of electrospun Gt/PCL/graphene membranes; (C) Mechanical properties of the electrospun Gt/PCL/graphene membranes. Left: tensile strength; middle: Young's modulus; right: strain at break. Data were expressed as mean $\pm \mathrm{SD}, \mathrm{n}=5$ per group. (D) Electrical conductivity of the electrospun $\mathrm{Gt} / \mathrm{PCL} / \mathrm{graphene}$ membranes. Data were expressed as mean $\pm \mathrm{SD}, \mathrm{n}=5$ per group. $0.3 \%, 0.5 \%, 0.8 \%$, and $\mathrm{I} .0 \%$ means different mass fractions of disordered $\mathrm{Gt} / \mathrm{PCL}$ membranes loaded with graphene.

Abbreviations: SEM, scanning electron microscope; Gt/PCL, gelatin/polycaprolactone.

$0.5 \%$, and $\mathrm{Gt} / \mathrm{PCL}$ with a graphene fraction of $1.0 \%$. This time, after 5 days of cell seeding, myocardial cells were strongly adhered and well spread on the scaffolds of $\mathrm{Gt} /$ PCL and Gt/PCL with a graphene fraction of $0.5 \%$. However, most of cells died when cultured on the scaffold with a graphene fraction of $1.0 \%$.
Furthermore, the same conclusion was indicated by the images shown in Figure 4. The cells were cultured with the scaffolds as listed above, and stained with DAPI, cTNT, and CX43 on the first, fifth, and ninth days of coculture. The percentage of apoptotic and necrotic cells was analyzed by fluorescence microscopy. It was clear 


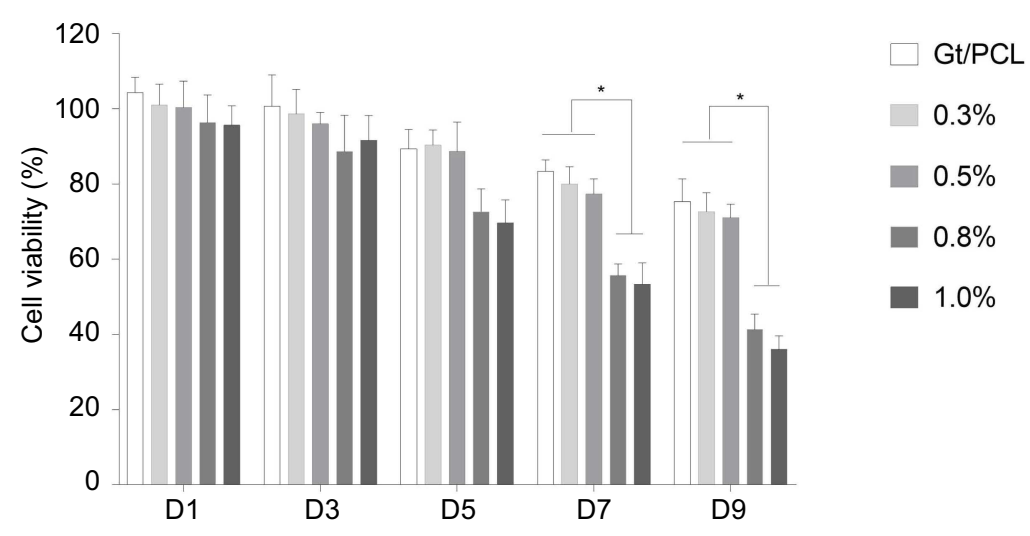

Figure 2 Cell apoptosis assay of different scaffolds measured by CCK-8. 0.3\%, 0.5\%, 0.8\%, and I.0\% means different mass fractions of disordered Gt/PCL membranes loaded with graphene. Cell viability was calculated as a percentage of the treated samples relative to untreated controls. The cell viability of all groups was measured every other day. Data are presented as mean \pm standard deviation, with $n=6 ; * P<0.05$.

Abbreviations: CCK-8, Cell Counting Kit-8; Gt/PCL, gelatin/polycaprolactone.

Gt/PL

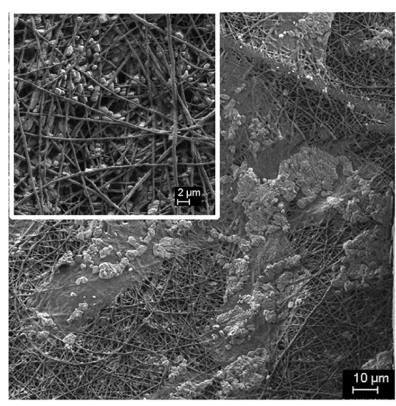

$0.5 \%$

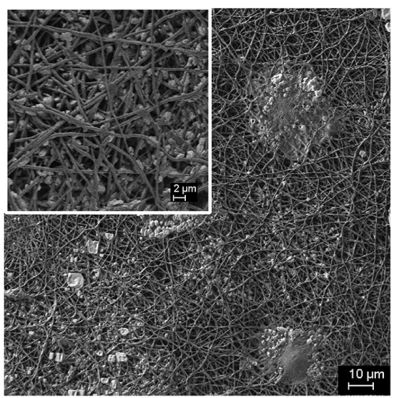

$1.0 \%$

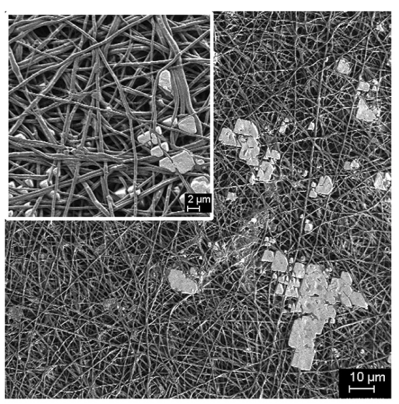

Figure 3 Cell adhesion and living condition on Gt/PCL/graphene membranes. Images of NRVCMs cells cultured on different GT/PCL/graphene scaffolds were captured by SEM at day 5 . Scale bars for the images and the insets are 10 and $2 \mu \mathrm{m}$, respectively. $0.5 \%$ and $1.0 \%$ means different mass fractions of disordered Gt/PCL membranes loaded with graphene.

Abbreviations: NRVCMs, neonatal rat ventricular cardiomyocytes; SEM, scanning electron microscope; Gt/PCL, gelatin/polycaprolactone.

from the results that pure Gt/PCL and Gt/PCL with a graphene mass fraction of $0.5 \%$ were nontoxic to myocardial cells.

\section{Gross observation}

The pure Gt/PCL membrane and those with $0.5 \%$ and $1.0 \%$ mass fractions of graphene were trimmed to a regular cylindrical shape and implanted subcutaneously into rats (Figure $5 \mathrm{~A}$ and $\mathrm{B}$ ). All implanted rats were raised in the same environment for the following 4, 8, and 12 weeks and carefully examined. No signs of weakness were detected. Scars on the back retained clean, and body weights of the animals were comparable. After 4, 8, and 12 weeks of implantation, constructs were harvested from rats (Figure 5C). Gross observation showed that all post-implantation constructs retained almost their original rounded shapes. There was no obvious difference detected among the three groups.

\section{Histological and immunohistological observations}

Histological staining was used to further support the aforementioned observations in terms of apoptosis. Histological analysis was performed on tissues obtained from the scaffold constructs to assess signs of acute toxicity. As shown in Figure 6, no cell necrosis or damage was detected in rats 4, 8 , or 12 weeks after implantation, clearly demonstrating that the nanofiber scaffolds did not cause damage to animals.

\section{Discussion}

The basic properties of Gt/PCL/graphene membranes, including morphological specificity, diameter, flexibility and electrical conductivity, are influenced by numerous factors and vary considerably with different mass fractions of graphene. ${ }^{23-26,30}$ According to earlier reports, electrospinning is a reliable and mature method to combine 


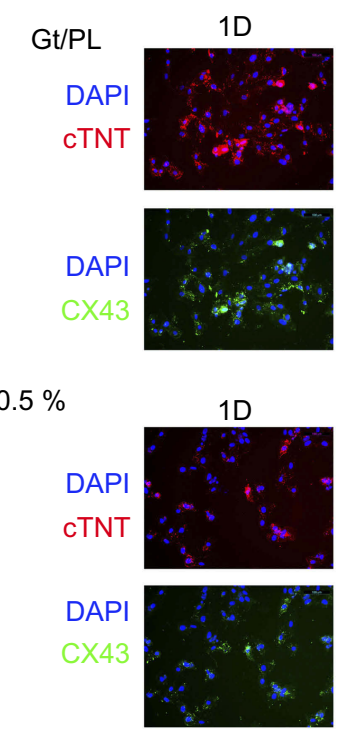

$1.0 \%$
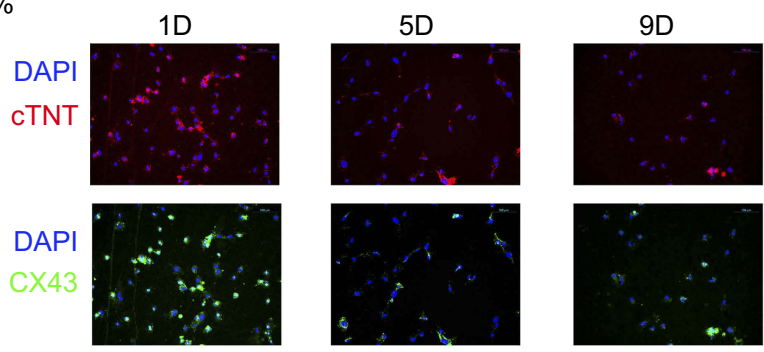

Figure 4 Immunohistochemical staining of the cell-scaffold constructs. Three different groups were set according to the mass fractions of disordered $\mathrm{Gt} / \mathrm{PCL}$ membranes loaded with graphene. Blue, DAPI for nucleic acid staining. Red, cTNT as specific marker of cardiomyocytes. Green, CX43 as another specific markers of cardiomyocytes. Scale bars: $100 \mu \mathrm{m}$.

Abbreviations: Gt/PCL, gelatin/polycaprolactone; DAPI, 4',6-diamidino-2-phenylindole; cTNT, cardiac troponin T; CX43, connexins 43.

gelatin and PCL. Gt/PCL, a new, synthetic nanofibrous membrane, represents a perfect scaffold for fabrication, with favorable mechanical and biological properties for tissue engineering. ${ }^{12,16,19,20,32,33}$ Here, graphene, which shows outstanding electrical conductivity and biological characteristics, was dissolved in Gt/PCL nanofibers. This procedure of conjugation improved the ability of the synthetic nanofibers to transmit electrical signals.

We initially focused on analysis of the nanomaterial's characteristics. As shown in the SEM images, larger fractions of graphene in Gt/PCL appeared to be associated with less stable nanofiber membranes. Different morphological features of beads could be detected in the image of Gt/PCL with $1.0 \%$ graphene. The presence of these beads indicated overdispersion of graphene, which could lead to damage or dysfunction of the scaffolds. The mechanical properties of the Gt/PCL/graphene membranes were calculated in terms of the Young's modulus. Our results indicated that the Gt/PCL/graphene membrane had
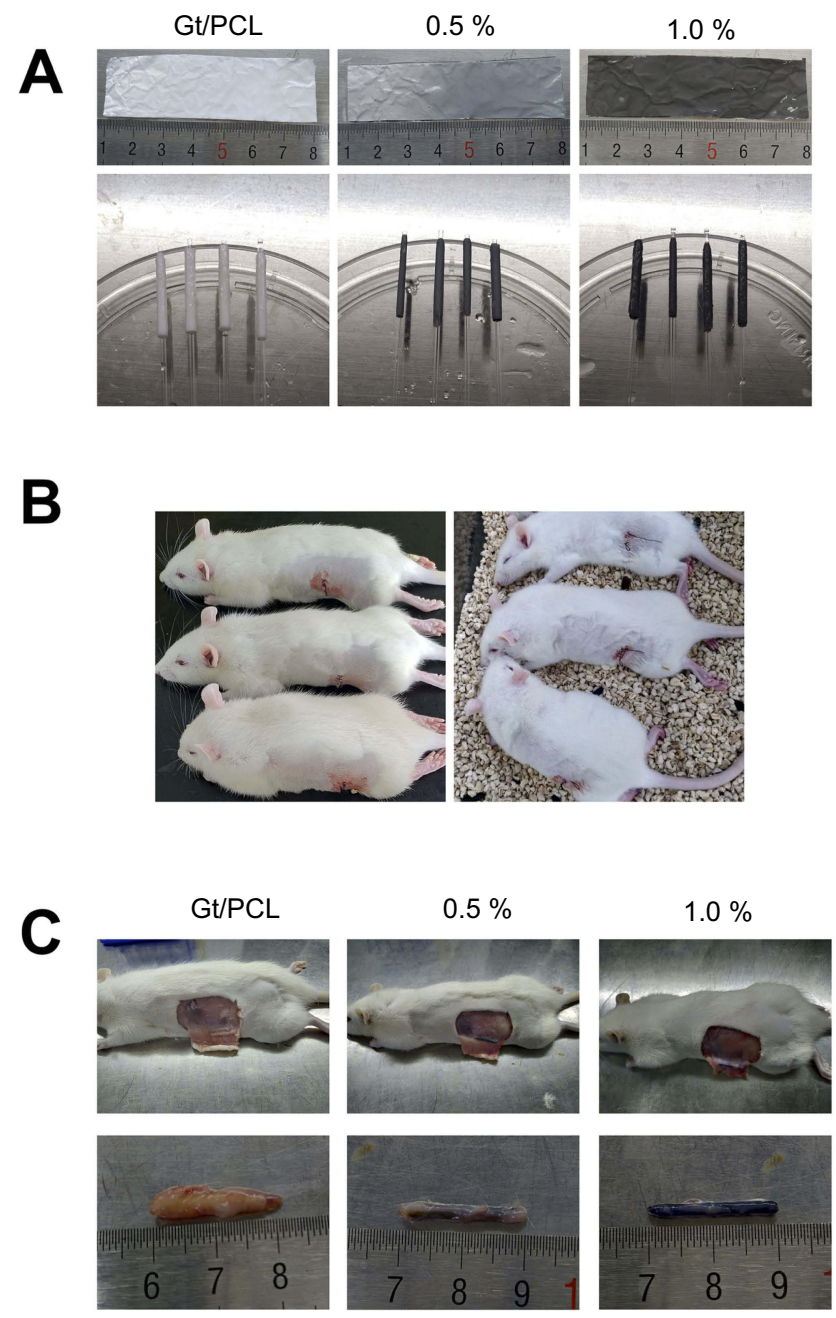

Figure 5 Gross view of implanting scaffold constructs. (A) Gross view of scaffold constructs. (B) Subcutaneous implantation in rats. (C) Scaffold constructs after 4 weeks in vivo implantation. $0.5 \%$ and $1.0 \%$ means different mass fractions of disordered $\mathrm{Gt} / \mathrm{PCL}$ membranes loaded with graphene.

Abbreviation: $\mathrm{Gt} / \mathrm{PCL}$, gelatin/polycaprolactone.

sufficient mechanical strength to endure systolic or muscular contractions. This is a key factor in the application of the nanomaterials, as a bypass tract rebinding electrical signals from atrium to ventricle should be implanted along with the myocardium. Three months after implantation, there was no damage to the nanofibers, and surrounding tissue cells had infiltrated the material of the Gt/PCL/ graphene membranes, together forming a cocultured organism with an adaptation to muscular adhesions.

However, it remained unknown whether the novel synthetic nanomaterial was toxic to normal myocardial cells or animals, as previous studies did not focus on toxicity assessments of the nanofibers. ${ }^{34,35}$ Nanomaterial toxicity is believed to be attributable to the release of graphene from the nanofibrous scaffolds and its accumulation within the body. ${ }^{36,37}$ In this study, we prepared five groups of Gt/PCL 

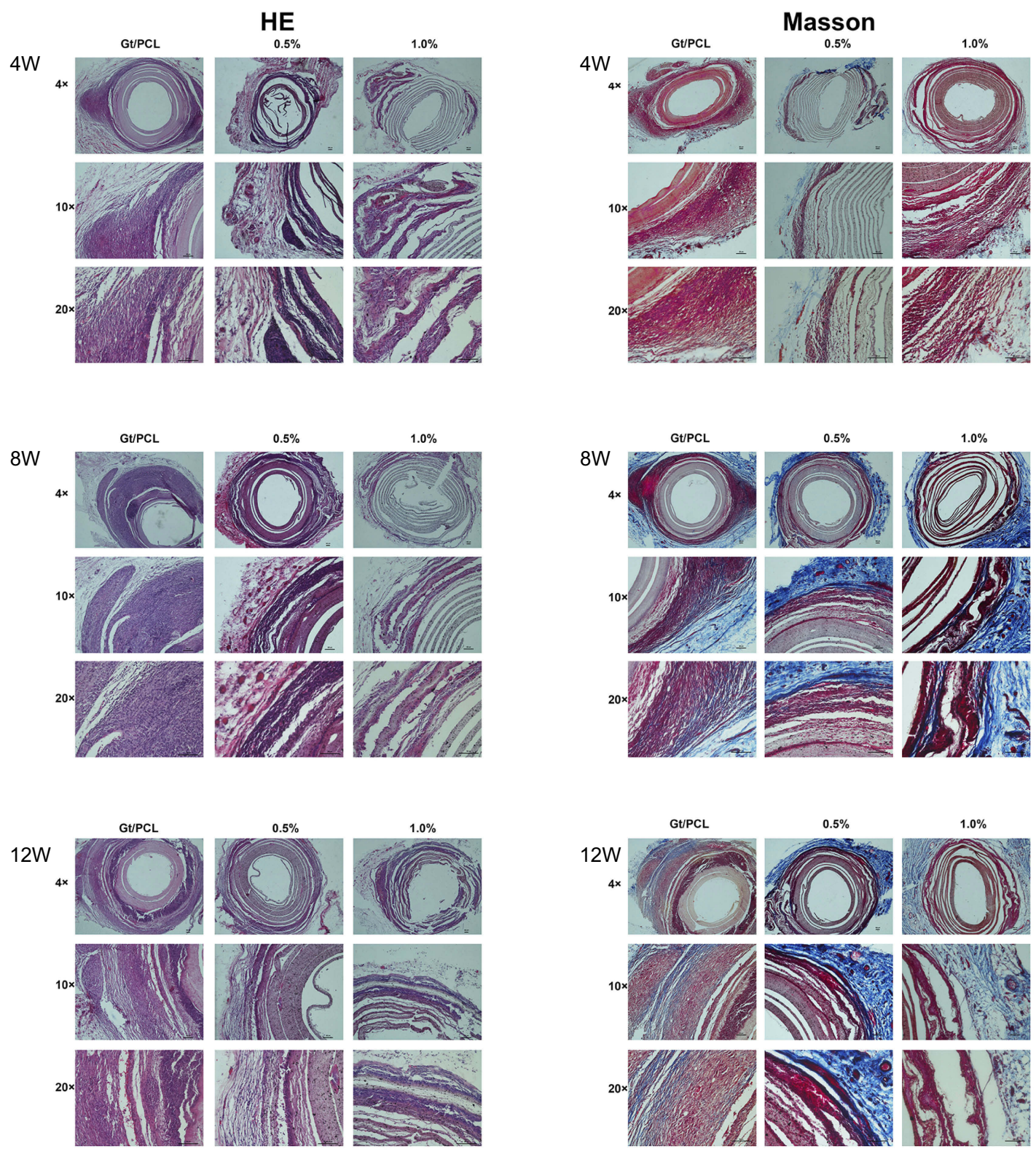

Figure 6 Histological images of scaffold constructs with H\&E and Masson's staining at 4, 8 and 12 weeks in vivo. No obvious cell necrosis or damage was detected in rats 4 , 8 , or 12 weeks after implantation. Scale bars: $100 \mu \mathrm{m} .0 .5 \%$ and $1.0 \%$ means different mass fractions of disordered $\mathrm{Gt} / \mathrm{PCL}$ membranes loaded with graphene. Abbreviations: $\mathrm{H \& E}$, hematoxylin and eosin; Gt/PCL, gelatin/polycaprolactone.

membranes with different graphene mass fractions $(0.0 \%$, $0.3 \%, 0.5 \%, 0.8 \%$, and $1.0 \%$ ). Based on the results of the CCK-8 assay, we concluded if the mass fraction of graphene was lower than $0.5 \%$, there would be no inhibition of the growth of NRVCMs. Thus, the pure Gt/PCL, $0.5 \%$ graphene, and $1.0 \%$ graphene membranes were chosen for the subsequent living condition experiments involving SEM imaging, and for immunohistochemical staining of the cell-scaffold constructs using DAPI for nucleic acid staining, and specific markers of cardiomyocytes, including cTNT and CX43. The results were consistent with the previously conducted CCK-8 assay, indicating that graphene was relatively nontoxic to myocardial cells at the dosage used. Excessive use of nanomaterials in the mixtures should be avoided, in view of environmental and toxicity concerns. Based on our comprehensive evaluations using CCK-8 assays, living condition images, and histological staining, we chose the $0.5 \%$ graphene membrane for subsequent tests of electrical signal transmission, owing to the relatively good coculture conditions and electrical conductivity observed for this membrane.

For comprehensive understanding of the toxicity mediated by the release of graphene administered via the implantation route, histopathological analysis of surrounding 
tissues of rats was required. ${ }^{38}$ The histopathology findings indicated that none of the Gt/PCL/graphene membranes caused toxicity over a short time-period, regardless of the dosage of graphene used. Furthermore, rats survived well in the subsequent 4,8 or 12 weeks, with no detectable adverse effects. Results from our in vitro and in vivo toxicity assessment collectively demonstrate that Gt/PCL/graphene membranes are relatively safe for use in myocardial cells and animals, further supporting their potential utility as novel nanofibers to transmit electrical conductivity.

\section{Conclusions}

Taken together, the results indicate that gelatin/PCL nanofibrous scaffolds loaded with graphene, with favorable electrical conductivity and biological properties, may be suitable for use in the treatment of AVB. These findings alleviate safety concerns and provide novel insights into the application of Gt/PCL/graphene membranes for local use in AVB, offering a solid foundation for subsequent specific studies.

\section{Acknowledgments}

This study was supported by the National Natural Science Foundation of China (grant number 81770332 and 81601622) and the Doctoral Innovation Fund of Shanghai Jiao Tong University School of Medicine (BXJ201842).

\section{Author contributions}

All authors contributed to data analysis, drafting or revising the article, gave final approval of the version to be published, and agree to be accountable for all aspects of the work.

\section{Disclosure}

The authors report no conflicts of interest in this work.

\section{References}

1. Vijayaraman P, Bordachar P, Ellenbogen KA. The continued search for physiological pacing: where are we now? J Am Coll Cardiol. 2017;69 (25):3099-3114. doi:10.1016/j.jacc.2017.05.005

2. Kusano KF, Satomi K. Diagnosis and treatment of cardiac sarcoidosis. Heart. 2016;102(3):184-190. doi:10.1136/heartjnl-2015-307877

3. Plotnikov AN, Sosunov EA, Qu J, et al. Biological pacemaker implanted in canine left bundle branch provides ventricular escape rhythms that have physiologically acceptable rates. Circulation. 2004;109(4):506-512.

4. Auffret V, Puri R, Urena M, et al. Conduction disturbances after transcatheter aortic valve replacement: current status and future perspectives. Circulation. 2017;136(11):1049-1069. doi:10.1161/ CIRCULATIONAHA.117.028352
5. Kutty S, Danford DA, Diller GP, Tutarel O. Contemporary management and outcomes in congenitally corrected transposition of the great arteries. Heart. 2018;104(14):1148-1155. doi:10.1136/heartjnl-2016-311032

6. Fujita B, Zimmermann WH. Engineered heart repair. Clin Pharmacol Ther. 2017;102(2):197-199. doi:10.1002/cpt.724

7. Lee AY, Mahler N, Best C, Lee YU, Breuer CK. Regenerative implants for cardiovascular tissue engineering. Transl Res. 2014;163(4):321-341. doi:10.1016/j.trsl.2014.01.014

8. Das A, Sinha M, Datta S, et al. Monocyte and macrophage plasticity in tissue repair and regeneration. Am J Pathol. 2015;185(10):25962606. doi:10.1016/j.ajpath.2015.06.001

9. Weinberger F, Mannhardt I, Eschenhagen T. Engineering cardiac muscle tissue: a maturating field of research. Circ Res. 2017;120 (9):1487-1500. doi:10.1161/CIRCRESAHA.117.310738

10. Ren J, Xu Q, Chen X, et al. Superaligned carbon nanotubes guide oriented cell growth and promote electrophysiological homogeneity for synthetic cardiac tissues. Adv mater. 2017;29(44). doi:10.1002/ adma.201700681.

11. Ma SP, Vunjak-Novakovic G. Tissue-Engineering for the study of cardiac biomechanics. J Biomech Eng. 2016;138(2):021010. doi:10.1115/1.4032997

12. Kishan AP, Cosgriff-Hernandez EM. Recent advancements in electrospinning design for tissue engineering applications: A review. J Biomed Materres A. 2017;105(10):2892-2905. doi:10.1002/jbm.a.36124

13. Schroder HC, Tolba E, Diehl-Seifert B, Wang X, Muller WE. Electrospinning of bioactive wound-healing nets. Prog Mol Subcell Biol. 2017;55:259-290. doi:10.1007/978-3-319-51284-6_8

14. Feng B, Wang S, Hu D, et al. Bioresorbable electrospun gelatin/ polycaprolactone nanofibrous membrane as a barrier to prevent cardiac postoperative adhesion. Acta Biomater. 2019;83:211-220. doi:10.1016/j.actbio.2018.10.022

15. He X, Feng B, Huang C, et al. Electrospun gelatin/polycaprolactone nanofibrous membranes combined with a coculture of bone marrow stromal cells and chondrocytes for cartilage engineering. Int $J$ Nanomedicine. 2015;10:2089-2099. doi:10.2147/IJN.S79461

16. Fu W, Liu Z, Feng B, et al. Electrospun gelatin/PCL and collagen/ PLCL scaffolds for vascular tissue engineering. Int J Nanomedicine. 2014;9:2335-2344. doi:10.2147/IJN.S61375

17. Wang W, He J, Feng B, et al. Aligned nanofibers direct human dermal fibroblasts to tenogenic phenotype in vitro and enhance tendon regeneration in vivo. Nanomedicine (Lond). 2016;11(9):1055-1072. doi:10.2217/nnm.16.24

18. Feng B, Tu H, Yuan H, Peng H, Zhang Y. Acetic-acid-mediated miscibility toward electrospinning homogeneous composite nanofibers of GT/PCL. Biomacromolecules. 2012;13(12):3917-3925. doi:10.1021/bm3009389

19. Yao R, He J, Meng G, Jiang B, Wu F. Electrospun PCL/Gelatin composite fibrous scaffolds: mechanical properties and cellular responses. $J$ Biomater Sci Polym Ed. 2016;27(9):824-838. doi:10.1080/09205063.2016.1160560

20. Strobel HA, Calamari EL, Beliveau A, Jain A, Rolle MW. Fabrication and characterization of electrospun polycaprolactone and gelatin composite cuffs for tissue engineered blood vessels. $J$ Biomed Mater Res B Appl Biomater. 2018;106(2):817-826. doi: $10.1002 / j b m . b .33871$

21. Zaretski AV, Root SE, Savchenko A, et al. Metallic nanoislands on graphene as highly sensitive transducers of mechanical, biological, and optical signals. Nano Lett. 2016;16(2):1375-1380. doi:10.1021/ acs.nanolett. $5 \mathrm{~b} 04821$

22. Ahadian S, Zhou Y, Yamada S, et al. Graphene induces spontaneous cardiac differentiation in embryoid bodies. Nanoscale. 2016;8 (13):7075-7084. doi:10.1039/c5nr07059g

23. Qian Y, Zhao X, Han Q, Chen W, Li H, Yuan W. An integrated multilayer 3D-fabrication of PDA/RGD coated graphene loaded PCL nanoscaffold for peripheral nerve restoration. Nat Commun. 2018;9 (1):323. doi:10.1038/s41467-017-02598-7 
24. Jalaja K, Sreehari VS, Kumar PRA, Nirmala RJ. Graphene oxide decorated electrospun gelatin nanofibers: fabrication, properties and applications. Mater sci eng C Mater biol appl. 2016;64:11-19. doi: $10.1016 /$ j.msec.2016.03.036

25. Nair M, Nancy D, Krishnan AG, Anjusree GS, Vadukumpully S, Nair SV. Graphene oxide nanoflakes incorporated gelatin-hydroxyapatite scaffolds enhance osteogenic differentiation of human mesenchymal stem cells. Nanotechnology. 2015;26(16):161001. doi:10.1088/09574484/26/16/161001

26. Liu H, Cheng J, Chen F, et al. Gelatin functionalized graphene oxide for mineralization of hydroxyapatite: biomimetic and in vitro evaluation. Nanoscale. 2014;6(10):5315-5322. doi:10.1039/c4nr00355a

27. Shin SR, Zihlmann C, Akbari M, et al. Reduced graphene OxideGelMA hybrid hydrogels as scaffolds for cardiac tissue engineering. Small. 2016;12(27):3677-3689. doi:10.1002/smll.201600178

28. Xing FY, Guan LL, Li YL, Jia CJ. Biosynthesis of reduced graphene oxide nanosheets and their in vitro cytotoxicity against cardiac cell lines of Catla catla. Environ Toxicol Pharmacol. 2016;48:110-115. doi:10.1016/j.etap.2016.09.022

29. Smith AST, Yoo H, Yi H, et al. Micro- and nano-patterned conductive graphene-PEG hybrid scaffolds for cardiac tissue engineering. Chem Commun (Camb). 2017;53(53):7412-7415. doi:10.1039/ c7cc01988b

30. Heidari M, Bahrami H, Ranjbar-Mohammadi M. Fabrication, optimization and characterization of electrospun poly(caprolactone)/gelatin/graphene nanofibrous mats. Mater sci eng C Mater biol appl. 2017;78:218-229. doi:10.1016/j.msec.2017.04.095
31. Contreras-Torres FF, Rodriguez-Galvan A, Guerrero-Beltran CE, et al. Differential cytotoxicity and internalization of graphene family nanomaterials in myocardial cells. Mater sci eng C Mater biol appl. 2017;73:633-642. doi:10.1016/j.msec.2016.12.080

32. Zhang Y, Ouyang H, Lim CT, Ramakrishna S, Huang ZM. Electrospinning of gelatin fibers and gelatin/PCL composite fibrous scaffolds. J Biomed Mater Res B Appl Biomater. 2005;72(1):156165. doi:10.1002/jbm.b.30128

33. Dvir T, Timko BP, Kohane DS, Langer R. Nanotechnological strategies for engineering complex tissues. Nat Nanotechnol. 2011;6 (1):13-22. doi:10.1038/nnano.2010.246

34. Takahashi T, Nagai T, Kanda M, et al. Regeneration of the Cardiac Conduction System by Adipose Tissue-Derived Stem Cells. Circ J. 2015;79(12):2703-2712. doi:10.1253/circj.CJ-15-0400

35. Kim T, Kahng YH, Lee T, Lee K, Kim DH. Graphene films show stable cell attachment and biocompatibility with electrogenic primary cardiac cells. Mol Cells. 2013;36(6):577-582. doi:10.1007/s10059013-0277-5

36. Nezakati T, Cousins BG, Seifalian AM. Toxicology of chemically modified graphene-based materials for medical application. Arch Toxicol. 2014;88(11):1987-2012. doi:10.1007/s00204-014-1361-0

37. Tonelli FM, Goulart VA, Gomes KN, et al. Graphene-based nanomaterials: biological and medical applications and toxicity. Nanomedicine (Lond). 2015;10(15):2423-2450. doi:10.2217/nnm.15.65

38. Waseem SH, Idrees MT, Croffie JM. Neuroenteric staining as a tool in the evaluation of pediatric motility disorders. Curr Gastroenterol Rep. 2015;17(8):30. doi:10.1007/s11894-015-0456-y

\section{Publish your work in this journal}

The International Journal of Nanomedicine is an international, peerreviewed journal focusing on the application of nanotechnology in diagnostics, therapeutics, and drug delivery systems throughout the biomedical field. This journal is indexed on PubMed Central, MedLine, CAS, SciSearch ${ }^{\mathbb{B}}$, Current Contents ${ }^{\mathbb{B}} /$ Clinical Medicine,
Journal Citation Reports/Science Edition, EMBase, Scopus and the Elsevier Bibliographic databases. The manuscript management system is completely online and includes a very quick and fair peer-review system, which is all easy to use. Visit http://www.dovepress.com/ testimonials.php to read real quotes from published authors. 